\title{
A Sharp Condition for Existence of an Inertial Manifold
}

\author{
Milan Miklavčič ${ }^{1}$
}

Received March 12, 1990

\begin{abstract}
It is shown that a perturbation argument that guarantees persistence of inertial (invariant and exponentially attracting) manifolds for linear perturbations of linear evolution equations applies also when the perturbation is nonlinear. This gives a simple but sharp condition for existence of inertial manifolds for semilinear parabolic as well as for some nonlinear hyperbolic equations. Fourier transform of the explicitly given equation for the tracking solution together with the Plancherel's theorem for Banach valued functions are used.
\end{abstract}

KEY WORDS: Inertial, invariant exponentially attracting manifolds; nonlinear evolution equations.

\section{INTRODUCTION}

In recent years it has been shown that solutions of many important PDE's approach exponentially to a flow on a smooth invariant finite dimensional manifold. See, for example, Henry [6], Foias et al. [4], Babin and Vishik [1], Mallet-Paret and Sell [7], Chow and Lu [3], Hale [5], Teman [9], and references therein. The crucial part often lies in finding an invariant attracting manifold for the flow in a Hilbert space $X$ generated by

$$
u^{\prime}+A u=F(u)
$$

where $A$ is a sectorial operator [6] in $X$ and $F$ is such that for some $\alpha \in[0,1), B \in \mathscr{B}\left(X^{\alpha}, X\right)$

$$
\|F(x)-F(y)\| \leqq\|B(x-y)\| \quad \text { for all } \quad x, y \in X^{x}
$$

\footnotetext{
${ }^{1}$ Department of Mathematics, Michigan State University, East Lansing, Michigan 48824-1027.
} 
here $\mathscr{B}\left(X^{\alpha}, X\right)$ denotes the space of bounded operators from $X^{\alpha}[6]$ into $X$. Various conditions that ensure existence of an inertial (invariant and attracting) manifold for (1.1) are known, however, since the problem seems to be a fundamental one, it would be nice to have optimal conditions. Here a condition that is sharp in a sense is presented.

Let $\lambda>0$ be such that

$$
\lambda+i \omega \quad \text { is in the resolvent set of } A, \rho(A), \quad \text { for all } \omega \in \mathbb{R}
$$

It is well known that $A$ has an invariant subspace $X_{1}$ which is the range of the projection associated with the spectral set in the half-plane $\operatorname{Re} z<\lambda$. $X_{1}$ is an inertial manifold for $u^{\prime}+A u=0$. If $\lambda+i \omega \in \rho(A-B)$ for all $\omega \in \mathbb{R}$, the same could be said for the equation $u^{\prime}+A u=B u$, and one way to ensure this is by requiring that

$$
\left\|B(A-\lambda-i \omega)^{-1}\right\|<1 \quad \text { for all } \omega \in \mathbb{R}
$$

since

$$
(A-B-\lambda-i \omega)^{-1}=(A-\lambda-i \omega)^{-1}\left(1-B(A-\lambda-i \omega)^{-1}\right)^{-1}
$$

In this paper it is proven that (1.2) is actually also sufficient for the existence of an inertial manifold for the nonlinear equation (1.1)no additional assumptions are needed. In spite of weaker and much simpler assumptions, the exponential atractivity result presented here (Theorem 4.1) is actually stronger then the one obtained by Babin and Vishik [1], Henry [6], Chow and Lu [3], and Foias et al. [4].

The paper is organized as follows. Assumptions, notation, and some well-known facts are presented in Section 2. Existence and some properties of the invariant manifold are derived in Section 3. In Section 4 exponential tracking is proven. Sections 3 and 4 are almost completely independent. In Section 5 it is shown how to modify assumptions so that the results of Sections 3 and 4 become applicable also to hyperbolic problems. A comparison of various assumptions is made in Section 6 .

\section{ASSUMPTIONS AND PRELIMINARIES}

The following is the list of all assumptions that will be in effect in Sections 3 and 4 :

(H1) $X$ is a complex Banach space.

(H2) There exists $M_{0} \in(0, \infty)$ such that if $f \in C(\mathbb{R} \backslash\{0\}, X)$ and $\|f(\cdot)\| \in L^{1}(\mathbb{R}) \cap L^{2}(\mathbb{R})$, then

$$
\int_{-\infty}^{-\infty}\|f(t)\|^{2} d t \leqslant M_{0} \int_{-\infty}^{\infty}\|\hat{f}(\omega)\|^{2} d \omega \leqslant M_{0}^{2} \int_{-\infty}^{\infty}\|f(t)\|^{2} d t
$$


where

$$
\hat{f}(\omega)=\frac{1}{\sqrt{2 \pi}} \int_{-\infty}^{\infty} e^{-i \omega t} f(t) d t \quad \text { for } \omega \in \mathbb{R}
$$

(H3) $A$ is a sectorial operator in $X, \alpha \in[0,1)$, and $X^{\alpha}$ is defined as usual (Ref. 6, p. 29).

(H4) $\lambda \in \mathbb{R}$ is such that $\lambda+i \omega$ is in the resolvent set of $A$ for all $\omega \in \mathbb{R}$.

(H5) $F: \mathbb{R} \times X^{\alpha} \rightarrow X$ is continuous and such that for some $B_{1}, \ldots, B_{m} \in$ $\mathscr{B}\left(X^{x}, X\right)$, we have that

$$
\|F(t, x)-F(t, y)\| \leqslant \sum_{j=1}^{m}\left\|B_{j}(x-y)\right\| \quad \text { for } \quad t \in \mathbb{R}, \quad x, y \in X^{\alpha}
$$

(H6) $\int_{-\infty}^{0}\left\|e^{\mu t} F(t, 0)\right\|^{2} d t<\infty$ for some $\mu<\lambda$.

(H7) $M_{0} \sum_{j=1}^{m} \sup _{\omega \in \mathbb{R}}\left\|B_{j}(A-\lambda-i \omega)^{-1}\right\|<1$.

Observe that if $X$ is any Hilbert space, then (H2) holds with $M_{0}=1$. Vagi [10] showed that if $M_{0}=1$, then $X$ has to be a Hilbert space. A slightly strengthened version of $\left(\mathrm{H}_{2}\right)$ would imply that $X$ would have to be homeomorphic to a Hilbert space. However, the intuitive argument presented in Section 1 suggests that $(\mathrm{H} 2)$ is probably not needed. (H2) is used only in the proofs of Lemmas 3.2 and 4.2 .

Various well-known consequences of the above assumptions and some definitions that are used in Sections 3 and 4 are now presented.

Let $\sigma(A)$ denote the spectrum of $A$. Choose $a<\inf \operatorname{Re} \sigma(A)$ and note that (Ref. 6, p. 29) $X^{\alpha}$ is equal to the domain of $(A-a)^{\alpha},\|x\|_{\alpha}=$ $\left\|(A-a)^{\alpha} x\right\|$ for $x \in X^{\alpha}$. Since (Ref. 6, p. 26)

$$
\left\|(A-a)^{\alpha}(A-\lambda-i \omega)^{-1}\right\| \leqslant \text { const. }\left\|(A-a)(A-\lambda-i \omega)^{-1}\right\|^{\alpha}\left\|(A-\lambda-i \omega)^{-1}\right\|^{1-\alpha}
$$

we have that

$$
\sup _{\omega \in \mathbb{R}}\left\|(A-a)^{x}(A-\lambda-i \omega)^{-1}\right\|<\infty
$$

Thus, by choosing $B_{0}=l(A-a)^{\alpha}$ with $l \in(0, \infty)$ small enough, we may assume that

$$
\rho(\lambda) \equiv M_{0} \sum_{j=0}^{m} c_{j}(\lambda)<1
$$

where

$$
c_{j}(\lambda)=\sup _{\omega \in \mathbb{R}}\left\|B_{j}(A-\lambda-i \omega)^{-1}\right\| \quad \text { for } \quad j=0,1, \ldots, m
$$


Since $A$ is sectorial we have that $\rho(\tilde{\lambda})<1$ whenever $|\lambda-\tilde{\lambda}|$ is small enough. Observe also that

$$
\|F(t, x)-F(t, y)\| \leqslant L\|x-y\|_{\alpha} \quad \text { for } \quad t \in \mathbb{R}, \quad x, y \in X^{\alpha}
$$

where

$$
L=\sum_{j=0}^{m}\left\|B_{j}(A-a)^{-x}\right\|
$$

Let

$$
\sigma_{1}=\{z \in \sigma(A) \mid \operatorname{Re} z<\lambda\}, \quad \sigma_{2}=\{z \in \sigma(A) \mid \operatorname{Re} z>\lambda\}
$$

Note that $\sigma(A)=\sigma_{1} \cup \sigma_{2}$ and that $\sigma_{1}$ is bounded. Let $P_{1} \in \mathscr{B}(X)$ be the projection associated with $\sigma_{1}, P_{2}=1-P_{1}, X_{i}=P_{i} X$ for $i=1,2$. Choose $\lambda_{1}, \lambda_{2} \in \mathbb{R}$ so that $\sup \operatorname{Re} \sigma_{1}<\lambda_{1}<\lambda<\lambda_{2}<\inf \operatorname{Re} \sigma_{2}$. As Henry (Ref. 6, p. 30), one obtains that

$$
\begin{aligned}
X_{1} & \subset \mathscr{D}(A), \quad A X_{1} \subset X_{1} \\
A_{1} & \equiv A \text { restricted to } X_{1}, \quad A_{1} \in \mathscr{B}\left(X_{1}\right) \\
P_{i} e^{-A t} & =e^{-A t} P_{i} \quad \text { for } \quad t \geqslant 0, \quad i=1,2 \\
e^{-A_{1} z} & =\sum_{n=0}^{\infty} \frac{\left(-A_{1} z\right)^{n}}{n !} \quad \text { for } \quad z \in \mathbb{C} \\
e^{-A_{1} t} x & =e^{-A t} x \quad \text { for } \quad x \in X_{1}, \quad t \geqslant 0
\end{aligned}
$$

and that there exists $M<\infty$ such that for all $x \in X$

$$
\begin{array}{rlrl}
\left\|e^{-A t}\right\| & \leqslant M e^{-a t}, & & t \geqslant 0 \\
\left\|e^{-A t} x\right\|_{\alpha} & \leqslant M t^{-\alpha} e^{-a t}\|x\|, & & t>0 \\
\left\|e^{-A_{1} t} P_{1} x\right\| & \leqslant M e^{-\lambda_{1} t}\|x\|, & t \leqslant 0 \\
\left\|e^{-A_{1} t} P_{1} x\right\|_{\alpha} & \leqslant M e^{-\lambda_{1} t}\|x\|, & t \leqslant 0 \\
\left\|e^{-A t} P_{2} x\right\| \leqslant M e^{-\lambda_{2} t}\|x\|, & t \geqslant 0 \\
\left\|e^{-A^{t}} P_{2} x\right\|_{\alpha} \leqslant M t^{-\alpha} e^{-\lambda_{2} t}\|x\|, & t>0
\end{array}
$$

\section{INVARIANT MANIFOLD}

For $\tau \in \mathbb{R}$ define $\mathscr{M}(\tau) \subset X$ as follows: $x \in \mathscr{M}(\tau)$ if and only if there exists $v \in C\left((-\infty, 0], X^{\alpha}\right)$ such that

$$
\begin{aligned}
& v(0)=x \\
& v(t)=e^{-A(t-T)} v(T)+\int_{T}^{t} e^{-A(t-s)} F(s+\tau, v(s)) d s \quad \text { for } \quad-\infty<T \leqslant t \leqslant 0 \\
& \int_{-\infty}^{0}\left\|e^{\lambda t} v(t)\right\|_{\alpha}^{p} d t<\infty \quad \text { for } p=1,2
\end{aligned}
$$


Note that for each $\tau \in \mathbb{R}, x \in X^{\alpha}$, there exists a unique $u \in C\left([\tau, \infty), X^{\alpha}\right)$ such that

$$
u(t)=e^{-A(t-\tau)} x+\int_{\tau}^{t} e^{-A(t-s)} F(s, u(s)) d s \quad \text { for } \quad t \geqslant \tau
$$

Therefore if $x \in \mathscr{M}(\tau)$, then there exists $u \in C\left(\mathbb{R}, X^{\alpha}\right)$ such that $u(\tau)=x$, $u(t) \in \mathscr{M}(t)$ for all $t \in \mathbb{R}$ and

$$
u(t)=e^{-A(t-T)} u(T)+\int_{T}^{t} e^{-A(t-s)} F(s, u(s)) d s \quad \text { for } \quad-\infty<T \leqslant t<\infty
$$

Thus, $\mathscr{M}$ is an invariant manifold. Obviously, if $F$ is periodic in or independent of the first variable, the same is true for $\mathscr{M}$. Some of the properties of $\mathscr{M}$ that are proved in the rest of this section are gathered in the following theorem.

Theorem 3.1. There exists a continuous $h: \mathbb{R} \times X_{1} \rightarrow X_{2} \cap X^{\alpha}$ such that

$$
\mathscr{M}(\tau)=\left\{x+h(\tau, x) \mid x \in X_{1}\right\} \quad \text { for all } \tau \in \mathbb{R}
$$

Moreover, there exists $c<\infty$ such that

$$
\|h(\tau, x)-h(\tau, y)\|_{x} \leqslant c\|x-y\| \quad \text { for all } \tau \in \mathbb{R}, \quad x, y \in X_{1}
$$

Define a normed space $Y$ by

$$
\begin{aligned}
Y & =\left\{v \in C\left((-\infty, 0], X^{\alpha}\right) \mid \int_{-\infty}^{0}\left\|e^{\lambda t} v(t)\right\|_{\alpha}^{p} d t<\infty \quad \text { for } p=1,2\right\} \\
|v|_{Y} & =\sum_{j=0}^{m}\left(\int_{-\infty}^{0}\left\|e^{\lambda t} B_{j} v(t)\right\|^{2} d t\right)^{1 / 2} \quad \text { for } \quad v \in Y
\end{aligned}
$$

Following Chow and Lu [3], define $S: \mathbb{R} \times Y \times X_{1} \rightarrow Y$ by

$$
\begin{aligned}
S(\tau, v, x)(t)= & e^{-A_{1} t} x+\int_{0}^{t} e^{-A_{1}(t-s)} P_{1} F(s+\tau, v(s)) d s \\
& +\int_{-\infty}^{t} e^{-A(t-s)} P_{2} F(s+\tau, v(s)) d s
\end{aligned}
$$

for $\tau \in \mathbb{R}, v \in Y, x \in X_{1}, t \leqslant 0$. To see that $u=S(\tau, v, x) \in Y$, observe that

$$
\left\|e^{\lambda t} u(t)\right\|_{\alpha} \leqslant M e^{\left(\lambda-\lambda_{1}\right) t}\|x\|+M \int_{-\infty}^{0} K(t-s) \theta(s) d s
$$


where

$$
\begin{aligned}
\theta(s) & =e^{\lambda s}\|F(s+\tau, v(s))\| \leqslant L\left\|e^{\lambda s} v(s)\right\|_{\alpha}+e^{\lambda s}\|F(s+\tau, 0)\| \\
K(t) & = \begin{cases}e^{\left(\lambda-\lambda_{1}\right) t} & \text { if } t \leqslant 0 \\
t^{-\alpha} e^{\left(\lambda-\lambda_{2}\right) t} & \text { if } t>0\end{cases} \\
\theta & \in L^{1}(-\infty, 0) \cap L^{2}(-\infty, 0), \quad K \in L^{1}(\mathbb{R})
\end{aligned}
$$

The following observation will be come useful:

$$
u(t)=e^{-A(t-T)} u(T)+\int_{T}^{t} e^{-A(t-s)} F(s+\tau, v(s)) d s \quad \text { for } \quad-\infty<T \leqslant t \leqslant 0
$$

which follows from the following:

$$
\begin{gathered}
P_{1} u(t)=e^{-A_{1} t} x+\int_{0}^{t} e^{-A_{1}(t-s)} P_{1} F(s+\tau, v(s)) d s \\
e^{A_{1} t} P_{1} u(t)=x+\int_{0}^{t} e^{A_{1} s} P_{1} F(s+\tau, v(s)) d s \\
P_{1} u(t)=e^{-A_{1}(t-T)} P_{1} u(T)+\int_{T}^{t} e^{-A_{1}(t-s)} P_{1} F(s+\tau, v(s)) d s \\
P_{2} u(t)=\int_{-\infty}^{t} e^{-A(t-s)} P_{2} F(s+\tau, v(s)) d s \\
P_{2} u(t)=e^{-A(t-T)} P_{2} u(T)+\int_{T}^{t} e^{-A(t-s)} P_{2} F(s+\tau, \tau, v(s)) d s
\end{gathered}
$$

Lemma 3.2. $|S(\tau, u, x)-S(\tau, v, x)|_{Y} \leqslant \rho(\lambda)|u-v|_{Y}$ for $\tau \in \mathbb{R}, x \in X_{1}$, $u, v \in Y$.

Proof. Let $c=S(\tau, u, x)(0)-S(\tau, v, x)(0)$

$$
\begin{aligned}
& g(t)= \begin{cases}e^{\lambda t} e^{-A t} c & \text { if } t>0 \\
e^{\lambda t}(S(\tau, u, x)(t)-S(\tau, v, x)(t)) & \text { if } t \leqslant 0\end{cases} \\
& f(t)= \begin{cases}0 & \text { if } t>0 \\
e^{\lambda t}(F(\tau+t, u(t))-F(\tau+t, v(t))) & \text { if } t \leqslant 0\end{cases}
\end{aligned}
$$

Note that $g \in C\left(\mathbb{R}, X^{\alpha}\right)$,

$$
\|g(\cdot)\|_{\alpha},\|f(\cdot)\| \in L^{1}(\mathbb{R}) \cap L^{2}(\mathbb{R})
$$




$$
\begin{aligned}
g(t) & =\int_{0}^{t} e^{\lambda(t-s)} e^{-A_{1}(t-s)} P_{1} f(s) d s+\int_{-\infty}^{t} e^{\lambda(t-s)} e^{-A(t-s)} P_{2} f(s) d s, \quad t \leqslant 0 \\
c & =P_{2} c=\int_{-\infty}^{0} e^{-\lambda s} e^{A s} P_{2} f(s) d s
\end{aligned}
$$

For $\omega \in \mathbb{R}$, let

$$
\hat{g}(\omega)=\frac{1}{\sqrt{2 \pi}} \int_{-\infty}^{\infty} e^{-i \omega t} g(t) d t \quad \text { (Bochner integral in } X^{\alpha} \text { ) }
$$

A straightforward calculation gives that for all $\omega \in \mathbb{R}$,

$$
\begin{aligned}
& \sqrt{2 \pi} \hat{g}(\omega)-(A-\lambda+i \omega)^{-1} c \\
&=\int_{-\infty}^{0} e^{-i \omega t} g(t) d t \\
&=-\int_{-\infty}^{0} d s \int_{-\infty}^{s} d t e^{(\lambda-i \omega)(t-s)} e^{-A_{1}(t-s)} P_{1} e^{-i \omega s} f(s) \\
& \quad+\int_{-\infty}^{0} d s \int_{s}^{0} d t e^{(\lambda-i \omega)(t-s)} e^{-A(t-s)} P_{2} e^{-i \omega s} f(s) \\
&=\int_{-\infty}^{0}(A-\lambda+i \omega)^{-1} P_{1} e^{-i \omega s} f(s) d s \\
& \quad+\int_{-\infty}^{0}(A-\lambda+i \omega)^{-1}\left(1-e^{A s} e^{-s(\hat{\lambda}-i \omega)}\right) P_{2} e^{-i \omega s} f(s) d s \\
& \hat{g}(\omega)=(A-\lambda+i \omega)^{-1} \hat{f}(\omega)
\end{aligned}
$$

Since $B_{j} \in \mathscr{B}\left(X^{\alpha}, X\right)$ for $j=0,1, \ldots, m$, we have that $\widehat{B_{j}} \hat{g}=B_{j} \hat{g}$ and

$$
\begin{aligned}
& \int_{-\infty}^{0}\left\|B_{j} g(t)\right\|^{2} d t \leqslant \int_{-\infty}^{\infty}\left\|B_{j} g(t)\right\|^{2} d t \leqslant M_{0} \int_{-\infty}^{\infty}\left\|B_{j} \hat{g}(\omega)\right\|^{2} d \omega \\
& \quad \leqslant M_{0} c_{j}^{2} \int_{-\infty}^{\infty}\|\hat{f}(\omega)\|^{2} d \omega \leqslant M_{0}^{2} c_{j}^{2} \int_{-\infty}^{0}\|f(t)\|^{2} d t \leqslant M_{0}^{2} c_{j}^{2}|u-v|_{Y}^{2}
\end{aligned}
$$

which implies the conclusion.

Lemma 3.3. For each $x \in X_{1}, \tau \in \mathbb{R}$, there exists a unique $v \in Y$ so that $S(\tau, v, x)=v$. 
Proof. Uniqueness follows from Lemma 3.2. Define

$$
\begin{aligned}
v_{0} & =0, \quad v_{n+1}=S\left(\tau, v_{n}, x\right) \quad \text { for } n \geqslant 0 \\
r_{n}(s) & =e^{2 s}\left(F\left(\tau+s, v_{n+1}(s)\right)-F\left(\tau+s, v_{n}(s)\right)\right) \quad \text { for } n \geqslant 0, \quad s \leqslant 0
\end{aligned}
$$

Note that for $n \geqslant 0, t \leqslant 0$,

$$
\begin{aligned}
\left(\int_{-\infty}^{0}\left\|r_{n}(s)\right\|^{2} d s\right)^{1 / 2} \leqslant & \left|v_{n+1}-v_{n}\right|_{Y} \leqslant \rho^{n}\left|v_{1}\right|_{Y} \\
e^{\lambda t}\left(v_{n+2}(t)-v_{n+1}(t)\right)= & \int_{0}^{t} e^{\lambda(t-s)} e^{-A_{1}(t-s)} P_{1} r_{n}(s) d s \\
& +\int_{-\infty}^{t} e^{\lambda(t-s)} e^{-A(t-s)} P_{2} r_{n}(s) d s
\end{aligned}
$$

$\left\|e^{\lambda t}\left(v_{n+2}(t)-v_{n+1}(t)\right)\right\| \leqslant k_{1} \rho^{n}, \quad k_{1}=M\left|v_{1}\right|_{Y}\left(\left(\lambda-\lambda_{1}\right)^{-1 / 2}+\left(\lambda_{2}-\lambda\right)^{-1 / 2}\right)$

Choose $\varepsilon \in(0,1)$ so that $\varepsilon \geqslant \rho$ and fix $T \in(-\infty, 0)$. For $n \geqslant 0$, $t \in(T, 0]$, we obtain from $(3.1)$

$$
\begin{gathered}
v_{n+2}(t)-v_{n+1}(t)=e^{-A(t-T)}\left(v_{n+2}(T)-v_{n+1}(T)\right) \\
\quad+\int_{T}^{t} e^{-A(t-s)}\left(F\left(s+\tau, v_{n+1}(s)\right)-F\left(s+\tau, v_{n}(s)\right)\right) d s \\
\left\|v_{n+2}(t)-v_{n+1}(t)\right\|_{\alpha} \leqslant M(t-T)^{-\alpha} e^{-a(t-T)-\lambda T} k_{1} \varepsilon^{n} \\
\quad+M L \int_{T}^{t}(t-s)^{-\alpha} e^{-\alpha(t-s)}\left\|v_{n+1}(s)-v_{n}(s)\right\|_{\alpha} d s \\
\leqslant k_{2}(t-T)^{-\alpha} \varepsilon^{n}+\varepsilon k_{3} \int_{T}^{t}(t-s)^{-\alpha}\left\|v_{n+1}(s)-v_{n}(s)\right\|_{\alpha} d s
\end{gathered}
$$

where $k_{2}=M e^{(a-\lambda) T} k_{1}\left(1+e^{-a T}\right), \quad k_{3}=M L\left(1+e^{a T}\right) / \varepsilon$. Thus, for $n \geqslant 1$, $t \in(T, 0]$,

$$
\begin{aligned}
& \varepsilon^{-n+1}\left\|v_{n+1}(t)-v_{n}(t)\right\|_{\alpha} \leqslant k_{2} \sum_{j=1}^{n} k_{3}^{j-1} \frac{\Gamma(1-\alpha)^{j}}{\Gamma(j-j \alpha)}(t-T)^{j-j \alpha-1} \\
& +k_{3}^{n} \frac{\Gamma(1-\alpha)^{n}}{\Gamma(n-n \alpha)} \int_{T}^{t}(t-s)^{n-n \alpha-1} \varepsilon\left\|v_{1}(s)\right\|_{\alpha} d s
\end{aligned}
$$

and therefore there exists $k_{4}<\infty$ such that

$$
\left\|v_{n+1}(t)-v_{n}(t)\right\|_{\alpha} \leqslant \varepsilon^{n-1}(t-T)^{-\alpha} k_{4} \quad \text { for } n \geqslant 1, \quad t \in(T, 0]
$$


Therefore there exists $v \in C\left((-\infty, 0], X^{\alpha}\right)$ such that for all $T \in(-\infty, 0)$,

and this implies

$$
\lim _{n \rightarrow \infty} \sup _{T \leqslant t \leqslant 0}\left\|v(t)-v_{n}(t)\right\|_{\alpha}=0
$$

$$
\begin{gathered}
\int_{-\infty}^{0}\left\|e^{\lambda t} v(t)\right\|_{\alpha}^{2} d t<\infty \\
\int_{-\infty}^{0}\left\|e^{\lambda t}\left(v(t)-v_{n}(t)\right)\right\|_{\alpha}^{2} d t \leqslant\left(\frac{\rho^{n}}{1-\rho} \frac{\left|v_{1}\right|_{Y}}{l}\right)^{2} \quad \text { for } n \geqslant 0
\end{gathered}
$$

Since $\rho(\tilde{\lambda})<1$ and $v_{n}$ do not depend on $\tilde{\lambda}$ for $\lambda$ close to $\lambda$ we also have

$$
\int_{-\infty}^{0}\left\|e^{\lambda t} v(t)\right\|_{\alpha}^{2} d t<\infty
$$

and hence $v \in Y,\left|v-v_{n}\right|_{Y} \rightarrow 0$ as $n \rightarrow \infty$ and therefore $S(\tau, v, x)=v$.

Define $h: \mathbb{R} \times X_{1} \rightarrow X_{2} \cap X^{\alpha}$ as follows: choose $x \in X_{1}, \tau \in \mathbb{R}$, let $v \in Y$ be such that $S(\tau, v, x)=v$ and define

$$
h(\tau, x)=P_{2} v(0)=\int_{-\infty}^{0} e^{A s} P_{2} F(s+\tau, v(s)) d s=v(0)-x
$$

Note that if $F$ is bounded in $X$ and $\lambda_{2}>0$, then $h$ is bounded in $X^{\alpha}$.

Lemma 3.4. $\mathscr{M}(\tau)=\left\{x+h(\tau, x) \mid x \in X_{1}\right\}$ for all $\tau \in \mathbb{R}$.

Proof. If $x \in X_{1}, \tau \in \mathbb{R}$, and $v \in Y$ satisfies $S(\tau, v, x)=v$, then $x+h(\tau, x)=v(0)$, and in view of (3.1) we have that $x+h(\tau, x) \in \mathscr{M}(\tau)$.

If $v$ is as in the definition of $\mathscr{M}(\tau)$, then for $t \leqslant 0$,

$$
\begin{aligned}
& P_{1} v(0)=e^{A_{1} t} P_{1} v(t)+\int_{t}^{0} e^{A_{1} s} P_{1} F(s+\tau, v(s)) d s \\
& P_{1} v(t)=e^{-A_{1} t} P_{1} v(0)+\int_{0}^{t} e^{-A_{1}(t-s)} P_{1} F(s+\tau, v(s)) d s
\end{aligned}
$$

If $-\infty<T \leqslant t \leqslant 0$, then

$$
P_{2} v(t)=e^{-A(t-T)} P_{2} v(T)+\int_{T}^{t} e^{-A(t-s)} P_{2} F(s+\tau, v(s)) d s
$$

and since the integral converges as $T \rightarrow-\infty$, the limit of

$$
\left\|e^{-A(t-T)} P_{2} v(T)\right\| \leqslant M e^{-\lambda_{2} t} e^{\lambda T}\|v(T)\|
$$


as $T \rightarrow-\infty$ has to exist and since $v \in Y$ it has to be 0 . Therefore

$$
P_{2} v(t)=\int_{-\infty}^{t} e^{-A(t-s)} P_{2} F(s+\tau, v(s)) d s \quad \text { for } t \leqslant 0
$$

and hence $v=S\left(\tau, v, P_{1} v(0)\right), v(0)=P_{1} v(0)+h\left(\tau, P_{1} v(0)\right)$.

Lemma 3.5. There exists $c<\infty$ such that

$$
\|h(\tau, x)-h(\tau, y)\|_{\alpha} \leqslant c\|x-y\| \quad \text { for all } \tau \in \mathbb{R}, x, y \in X_{1}
$$

Proof. Choose $x, y \in X_{1}, \tau \in \mathbb{R}$ and let $u, v \in Y$ be such that

$$
S(\tau, u, x)=u, \quad S(\tau, v, y)=v
$$

$d_{1}, d_{2}, \ldots$, denote various constants - independent of $\tau, x, y$. Note that

$$
\begin{aligned}
& u-v= S(\tau, u, y)-S(\tau, v, y)+e^{-A_{1} t}(x-y) \\
&|u-v|_{Y} \leqslant \rho|u-v|_{Y}+d_{1}\|x-y\| \\
&|u-v|_{Y} \leqslant d_{2}\|x-y\| \\
& e^{\lambda t}(u(t)-v(t))= e^{\lambda t} e^{-A_{1} t}(x-y)+\int_{0}^{t} e^{\lambda(t-s)} e^{-A_{1}(t-s)} P_{1} r(s) d s \\
&+\int_{-\infty}^{t} e^{\lambda(t-s)} e^{-A(t-s)} P_{2} r(s) d s
\end{aligned}
$$

where $r(t)=e^{\lambda t}(F(t+\tau, u(t))-F(t+\tau, v(t)))$ for $t \leqslant 0$. Since $\int_{-\infty}^{0}\|r(t)\|^{2} d t$ $\leqslant|u-v|_{Y}^{2}$

$$
\begin{aligned}
e^{\lambda t}\|u(t)-v(t)\| \leqslant & M\|x-y\|+M \int_{t}^{0} e^{\left(\lambda-\lambda_{1}\right)(t-s)}\|r(s)\| d s \\
& +M \int_{-\infty}^{t} e^{\left(\lambda-\lambda_{2}\right)(t-s)}\|r(s)\| d s \\
\leqslant & M\|x-y\|+d_{3}|u-v|_{Y} \leqslant d_{4}\|x-y\| \quad \text { for } \quad t \leqslant 0
\end{aligned}
$$

Equation (3.1) implies that for $-\infty<T<t \leqslant 0$,

$$
\begin{aligned}
u(t)-v(t)= & e^{-A(t-T)}(u(T)-v(T)) \\
& +\int_{T}^{t} e^{-A(t-s)}(F(s+\tau, u(s))-F(s+\tau, v(s))) d s \\
\|u(t)-v(t)\|_{\alpha} \leqslant & M(t-T)^{-\alpha} e^{-a(t-T)-\lambda T} d_{4}\|x-y\| \\
& +M L \int_{T}^{t}(t-s)^{-\alpha} e^{-a(t-s)}\|u(s)-v(s)\|_{\alpha} d s
\end{aligned}
$$


which implies [see (3.2)] that for some $d_{5}$ we have

$$
\|u(t)-v(t)\|_{\alpha} \leqslant\|x-y\|(t-T)^{-\alpha} d_{5} \quad \text { for } \quad t \in(T, 0]
$$

and since $h(\tau, x)-h(\tau, y)=u(0)-v(0)-x+y$, we are done.

Lemma 3.6. $h: \mathbb{R} \times X_{1} \rightarrow X^{\alpha}$ is continuous.

Remark. Inequality (3.3) below can sometimes imply more regularity of $h$.

Proof of Lemma 3.6. Fix $\tau \in \mathbb{R}, x \in X_{1}$. Take $u \in C\left(\mathbb{R}, X^{\infty}\right)$ such that $u(\tau)=x+h(\tau, x), u(t) \in \mathscr{M}(t)$ for $t \in \mathbb{R}$ and for $-\infty<T \leqslant t<\infty$,

$$
u(t)=e^{-A(t-T)} u(T)+\int_{T}^{t} e^{-A(t-s)} F(s, u(s)) d s
$$

For $\sigma \in \mathbb{R}, y \in X_{1}$ we have

$$
\begin{aligned}
h(\sigma, y)-h(\tau, x)= & h(\sigma, y)-h(\sigma, x)+h\left(\sigma, P_{1} u(\tau)\right)-h\left(\sigma, P_{1} u(\sigma)\right) \\
& +P_{2} u(\sigma)-P_{2} u(\tau) \\
\|h(\sigma, y)-h(\tau, x)\|_{\alpha} \leqslant & c\|y-x\|+c\left\|P_{1}\right\|\|u(\tau)-u(\sigma)\| \\
& +\left\|P_{2}\right\|\|u(\sigma)-u(\tau)\|_{\alpha}
\end{aligned}
$$

Therefore $\|h(\sigma, y)-h(\tau, x)\|_{x} \rightarrow 0$ as $\sigma \rightarrow \tau, y \rightarrow x$.

\section{EXPONENTIAL TRACKING}

Choose any $\tau \in \mathbb{R}, u \in C\left([\tau, \infty), X^{\alpha}\right)$ such that

$$
u(t)=e^{-A(t-\tau)} u(\tau)+\int_{\tau}^{t} e^{-A(t-s)} F(s, u(s)) d s \quad \text { for } \quad t \geqslant \tau
$$

The purpose of this section is to prove the following.

Theorem 5.1. There exists a unique $v \in C\left(\mathbb{R}, X^{\alpha}\right)$ such that

$$
\begin{array}{cc}
v(t)=e^{-A(t-T)} v(T)+\int_{T}^{t} e^{-A(t-s)} F(s, v(s)) d s & \text { for } \quad-\infty<T \leqslant t<\infty \\
\int_{\tau}^{\infty}\left\|e^{\lambda t}(u(t)-v(t))\right\|_{\alpha}^{p} d t+\int_{-\infty}^{\tau}\left\|e^{\lambda t} v(t)\right\|_{\alpha}^{p} d t<\infty & \text { for } \quad p=1,2
\end{array}
$$


Moreover, $v(t) \in \mathscr{M}(t)$ for all $t \in \mathbb{R}$ and there exists $C \in[0, \infty)$ which depends only on $M, \lambda, \lambda_{1}, \lambda_{2}, \alpha, L, l, \rho(\lambda)$, such that

$$
e^{\lambda t}\|v(t)-u(t)\|_{\alpha} \leqslant C e^{\lambda T} \inf _{x \in \mathscr{M}(T)}\|x-u(T)\|_{\alpha} \quad \text { for all } \tau \leqslant T \leqslant t<\infty
$$

Theorem 3.1 gives a bound for

$$
P_{2} u(t)-h\left(t, P_{1} u(t)\right)=P_{2}(u(t)-v(t))+h\left(t, P_{1} v(t)\right)-h\left(t, P_{1} u(t)\right)
$$

Define $u(t)=e^{-\lambda_{1}(t-\tau)} u(\tau)$ for $t<\tau$ and let

$$
\begin{aligned}
Z= & \left\{\phi \in C\left(\mathbb{R}, X^{\alpha}\right) \mid \int_{-\infty}^{\infty}\left\|e^{\lambda t} \phi(t)\right\|_{\alpha}^{p} d t<\infty \quad \text { for } p=1,2\right\} \\
|\phi|_{z}= & \sum_{j=0}^{m}\left(\int_{-\infty}^{\infty}\left\|e^{\lambda t} B_{j} \phi(t)\right\|^{2} d t\right)^{1 / 2} \text { for } \phi \in Z \\
w(t)= & -u(t)+e^{-A_{1}(t-\tau)} P_{1} u(\tau)+\int_{-\infty}^{t} e^{-A(t-s)} P_{2} F(s, u(s)) d s \\
& -\int_{t}^{\tau} e^{-A_{1}(t-s)} P_{1} F(s, u(s)) d s \text { for } t \leqslant \tau \\
w(t)= & e^{-A(t-\tau)} w(\tau)=e^{-A(t-\tau)} P_{2} w(\tau) \text { for } t>\tau
\end{aligned}
$$

Observe that $w \in Z$. Define $R: Z \rightarrow Z$ by

$$
\begin{aligned}
(R \phi)(t)= & w(t)+\int_{-\infty}^{t} e^{-A(t-s)} P_{2}(F(s, \phi(s)+u(s))-F(s, u(s))) d s \\
& -\int_{t}^{\infty} e^{-A_{1}(t-s)} P_{1}(F(s, \phi(s)+u(s))-F(s, u(s))) d s
\end{aligned}
$$

Lemma 4.2. $|R \phi-R \psi|_{z} \leqslant \rho(\lambda)|\phi-\psi|_{z}$ for $\phi, \psi \in Z$.

Proof. For $t \in \mathbb{R}$ let

$$
\begin{aligned}
& g(t)=e^{\lambda t}((R \phi)(t)-(R \psi)(t)) \\
& f(t)=e^{\lambda t}(F(t, \phi(t)+u(t))-F(t, \psi(t)+u(t)))
\end{aligned}
$$

As in the proof of Lemma 3.2,

$$
\begin{aligned}
\hat{g}(\omega) & =(A-\lambda+i \omega)^{-1} \hat{f}(\omega) \quad \text { for } \omega \in \mathbb{R} \\
\int_{-\infty}^{\infty}\left\|B_{j} g(t)\right\|^{2} d t & \leqslant M_{0} \int_{-\infty}^{\infty}\left\|B_{j} \hat{g}(\omega)\right\|^{2} d \omega \leqslant M_{0}^{2} c_{j}^{2} \int_{-\infty}^{\infty}\|f(t)\|^{2} d t \\
& \leqslant M_{0}^{2} c_{j}^{2}|\phi-\psi|_{z}^{2}
\end{aligned}
$$


Lemma 4.3. There exists a unique $\Theta \in Z$ such that $R \Theta=\Theta$. Moreover, there exists $C \in[0, \infty)$ which depends only on $M, \lambda, \lambda_{1}, \lambda_{2}, \alpha, L, l, \rho(\lambda)$, such that

$$
e^{\lambda t}\|\Theta(t)\|_{\alpha} \leqslant C e^{\lambda T} \inf _{x \in \mathscr{M}(T)}\|x-u(T)\|_{\alpha} \quad \text { whenever } \quad \tau \leqslant T \leqslant t<\infty
$$

Proof. Uniqueness follows from Lemma 4.2. Let $\phi_{0}=0, \phi_{n+1}=R \phi_{n}$ for $n \geqslant 0$. Note that for $t \in \mathbb{R}, n \geqslant 0$,

$$
\begin{aligned}
e^{\lambda t}\left(\phi_{n+2}(t)-\phi_{n+1}(t)\right)= & \int_{-\infty}^{t} e^{\lambda(t-s)} e^{-A(t-s)} P_{2} r_{n}(s) d s \\
& -\int_{r}^{\infty} e^{\lambda(t-s)} e^{-A_{1}(t-s)} P_{1} r_{n}(s) d s \\
r_{n}(t) & =e^{\lambda t}\left(F\left(t, \phi_{n+1}(t)+u(t)\right)-F\left(t, \phi_{n}(t)+u(t)\right)\right)
\end{aligned}
$$

Let $f_{n}(t)=e^{\lambda t}\left\|\phi_{n+1}(t)-\phi_{n}(t)\right\|_{\alpha}$ and

$$
K(t)= \begin{cases}M L e^{\left(\lambda-\lambda_{1}\right) t} & \text { if } t \leqslant 0 \\ M L t^{-x} e^{\left(\lambda-\lambda_{2}\right) t} & \text { if } t>0\end{cases}
$$

and note that

$$
f_{n+1}(t) \leqslant \int_{-\infty}^{\infty} K(t-s) f_{n}(s) d s \quad \text { for } \quad n \geqslant 0, \quad t \in \mathbb{R}
$$

Define $K_{1}=K, K_{j+1}=K * K_{j}$ for $j \geqslant 1$ and note that

$$
f_{n+j} \leqslant K_{j} * f_{n} \quad \text { for } n \geqslant 0, \quad j \geqslant 1
$$

Choose an integer $N \geqslant 1$ such that $2 N(1-\alpha)>1$. Young's inequality (Ref. 6, p. 34) gives

$$
K_{n} \in L^{q_{n}}(\mathbb{R}), \quad q_{n}=2 N /(2 N-n) \quad \text { for } \quad 1 \leqslant n \leqslant 2 N
$$

Thus, $K_{N} \in L^{2}(\mathbb{R})$ and for $n \geqslant 0$,

$$
\begin{aligned}
& \left\|f_{n+N}\right\|_{\infty} \leqslant\left\|K_{n}\right\|_{2}\left\|f_{n}\right\|_{2} \leqslant\left\|K_{N}\right\|_{2} l^{-1}\left|\phi_{n+1}-\phi_{n}\right|_{z} \\
& \left\|f_{n+N}\right\|_{\infty} \leqslant K_{N} \|_{2} l^{-1}\left|\phi_{1}\right|_{z} \rho^{n}
\end{aligned}
$$

Therefore, there exist $\Theta \in C\left(\mathbb{R}, X^{\alpha}\right), d \in \mathbb{R}$ such that

$$
e^{\lambda t}\left\|\Theta(t)-\phi_{n}(t)\right\|_{\alpha} \leqslant d \rho^{n-N} \quad \text { for } \quad n \geqslant N, \quad t \in \mathbb{R}
$$


Using the facts that we can replace $\lambda$ with $\lambda$ provided that $|\lambda-\bar{\lambda}|$ is small enough and that this change does not effect $\phi_{n}$ and hence $\Theta$, we see that there exist $d_{1}<\infty, \varepsilon<1, \delta>0$, such that

$$
e^{\lambda t}\left\|\Theta(t)-\phi_{n}(t)\right\|_{\alpha} \leqslant d_{1} \varepsilon^{n} e^{-\delta|t|} \quad \text { for } \quad t \in \mathbb{R}, \quad n \geqslant N
$$

Thus $\Theta \in Z,\left|\Theta-\phi_{n}\right|_{z} \rightarrow 0$ as $n \rightarrow \infty$ and therefore $R \Theta=\Theta$.

To prove the moreover part, choose any $T \in[\tau, \infty), x \in \mathscr{M}(T)$. Let $\psi \in Y$ be such that $S\left(T, \psi, P_{1} x\right)=\psi$, hence $x=\psi(0)$. Define

$$
\psi_{0}(t)= \begin{cases}\psi(t-T)-u(t) & \text { for } t \leqslant T \\ 0 & \text { for } t>T\end{cases}
$$

$\psi_{0}$ is in general not continuous, however, $\psi_{1} \equiv R \psi_{0}$ can clearly be evaluated, and a long but straightforward calculation gives

$$
\psi_{1}(t)-\psi_{0}(t)= \begin{cases}e^{-A_{1}(t-T)} P_{1}(u(T)-x) & \text { if } t \leqslant T \\ e^{-A(t-T)} P_{2}(x-u(T)) & \text { if } t>T\end{cases}
$$

and thus $\psi_{1} \in Z$. Define $\psi_{n+1}=R \psi_{n}$ also for $n \geqslant 1$.

Since $\left|\phi_{n}-\Theta\right|_{z} \rightarrow 0$ as $n \rightarrow \infty$, Lemma 4.2 implies

$$
\left|\Theta-\psi_{n}\right|_{z} \leqslant \rho^{n-1}\left|\psi_{2}-\psi_{1}\right|_{z} /(1-\rho) \quad \text { for } n \geqslant 1
$$

For $n \geqslant 0, t \in \mathbb{R}$, define $g_{n}(t)=e^{\lambda t}\left\|\psi_{n+1}(t)-\psi_{n}(t)\right\|_{\alpha}$. As above,

$$
\begin{aligned}
& g_{n+j} \leqslant K_{j} * g_{n} \quad \text { for } n \geqslant 0, \quad j \geqslant 1 \\
& \left\|g_{n+N}\right\|_{\infty} \leqslant\left\|K_{N}\right\|_{2}\left\|g_{n}\right\|_{2} \leqslant\left\|K_{N}\right\|_{2} l^{-1}\left|\psi_{n+1}-\psi_{n}\right|_{z} \\
& \leqslant\left\|K_{N}\right\|_{2} l^{-1}\left|\psi_{2}-\psi_{1}\right|_{z} \rho^{n-1} \quad \text { for } n \geqslant 1 \\
& \left\|g_{n}\right\|_{\infty} \leqslant\left\|K_{N}\right\|_{2} L l^{-1}\left\|g_{1}\right\|_{2} \rho^{n-N-1} \\
& \leqslant\left\|K_{N}\right\|_{2}\|K\|_{1} L l^{-1}\left\|g_{0}\right\|_{2} \rho^{n-N-1} \quad \text { for } \quad n \geqslant N+1 \\
& e^{\lambda t}\left\|\psi_{j}(t)-\psi_{n}(t)\right\|_{\alpha} \leqslant\left\|K_{N}\right\|_{2}\|K\|_{1} L l^{-1}(1-\rho)^{-1}\left\|g_{0}\right\|_{2} \rho^{n-N-1} \\
& \text { for } \quad N+1 \leqslant n \leqslant j, \quad t \in \mathbb{R} \\
& e^{\lambda t}\left\|\Theta(t)-\psi_{n}(t)\right\|_{\alpha} \leqslant\left\|K_{N}\right\|_{2}\|K\|_{1} L l^{-1}(1-\rho)^{-1}\left\|g_{0}\right\|_{2} \rho^{n-N-1} \\
& \text { for } n \geqslant N+1, \quad t \in \mathbb{R}
\end{aligned}
$$

For $t>T$ we have

$$
\begin{aligned}
e^{\lambda t}\|\Theta(t)\|_{\alpha} \leqslant & e^{\lambda t}\left\|\Theta(t)-\psi_{N+1}(t)\right\|_{\alpha}+g_{0}(t)+g_{1}(t)+\cdots+g_{N}(t) \\
\leqslant & \left\|K_{N}\right\|_{2}\|K\|_{1} L l^{-1}(1-\rho)^{-1}\left\|g_{0}\right\|_{2} \\
& +\left(1+\|K\|_{1}+\cdots+\|K\|_{1}^{N}\right)\left\|g_{0}\right\|_{\infty}
\end{aligned}
$$


Evaluation of $\left\|g_{0}\right\|_{2},\left\|g_{0}\right\|_{\infty}$, gives

$$
e^{\lambda t}\|\Theta(t)\|_{\alpha} \leqslant C e^{\lambda T}\|x-u(T)\|_{\alpha} \quad \text { for } \quad t \geqslant T
$$

where

$$
e^{\lambda t}\|\Theta(t)\|_{\alpha} \leqslant C e^{\lambda T}\|x-u(T)\|_{\alpha} \quad \text { for } \quad t \geqslant T
$$

where

$$
\begin{aligned}
C= & \left\|K_{N}\right\|_{2}\|K\|_{1} L l^{-1}(1-\rho)^{-1} M \sqrt{1 /\left[2\left(\lambda-\lambda_{1}\right)\right]+1 /\left[2\left(\lambda_{2}-\lambda\right)\right]} \\
& +M\left(1+\|K\|_{1}+\cdots+\|K\|_{1}^{N}\right)
\end{aligned}
$$

Proof of Theorem 4.1. If $\Theta \in Z$ is such that $R \Theta=\Theta$ and $v=\Theta+u$, then a long but obvious calculation shows that this $v$ has the desired properties.

Suppose that we have $v$ as in Theorem 4.1. Obviously $v-u \in Z$. We show that $v-u=R(v-u)$ and hence Lemma 4.3 implies uniqueness.

For $-\infty<T \leqslant t<\infty$ we have

$$
\begin{aligned}
P_{2}(v(t)-u(t))= & -P_{2} u(t)+e^{-A(t-T)} P_{2} v(T) \\
& +\int_{T}^{t} e^{-A(t-s)} P_{2}(F(s, v(s))-F(s, u(s))) d s \\
& +\int_{T}^{t} e^{-A(t-s)} P_{2} F(s, u(s)) d s
\end{aligned}
$$

letting $T \rightarrow-\infty$, we obtain (as in the proof of Lemma 3.4)

$$
\begin{aligned}
P_{2}(v(t)-u(t))= & -P_{2} u(t)+\int_{-\infty}^{t} e^{-A(t-s)} P_{2} F(s, u(s)) d s \\
& +\int_{-\infty}^{t} e^{-A(t-s)} P_{2}(F(s, v(s))-F(s, u(s))) d s
\end{aligned}
$$

If $\tau \leqslant t \leqslant T$, then

$$
\begin{aligned}
P_{1}(v(T)-u(T))= & e^{-A_{1}(T-t)} P_{1}(v(t)-u(t)) \\
& +\int_{t}^{T} e^{-A_{1}(T-s)} P_{1}(F(s, v(s))-F(s, u(s))) d s \\
P_{1}(v(t)-u(t))= & e^{-A_{1}(t-T)} P_{1}(v(T)-u(T)) \\
& -\int_{t}^{T} e^{-A_{1}(t-s)} P_{1}(F(s, v(s))-F(s, u(s))) d s
\end{aligned}
$$


letting $T \rightarrow \infty$ we obtain that for $t \geqslant \tau$,

$$
P_{1}(v(t)-u(t))=-\int_{t}^{\infty} e^{-A_{1}(t-s)} P_{1}(F(s, v(s))-F(s, u(s))) d s
$$

If $t \leqslant \tau$, then

$$
\begin{aligned}
P_{1}(v(t)-u(t))= & -P_{1} u(t)+e^{-A_{1}(t-\tau)} P_{1}(v(\tau)-u(\tau))+e^{-A_{1}(t-\tau)} P_{1} u(\tau) \\
& -\int_{t}^{\tau} e^{-A_{1}(t-s)} P_{1} F(s, v(s)) d s
\end{aligned}
$$

and (4.3) implies

$$
\begin{aligned}
= & -P_{1} u(t)+e^{-A_{1}(t-\tau)} P_{1} u(\tau)-\int_{t}^{\tau} e^{-A_{1}(t-s)} P_{1} F(s, u(s)) d s \\
& -\int_{t}^{\infty} e^{-A_{1}(t-s)} P_{1}(F(s, v(s))-F(s, u(s))) d s \\
= & P_{1} w(t)-\int_{t}^{\infty} e^{-A_{1}(t-s)} P_{1}(F(s, v(s))-F(s, u(s))) d s
\end{aligned}
$$

this, (4.2), and (4.3) imply that $v-u=R(v-u)$.

\section{HYPERBOLIC EXTENSION}

The assumption used so far that $A$ is a sectorial operator can be weakened by requiring that $-A$ is the generator of a strongly continuous semigroup and thus the theory becomes applicable to hyperbolic problems. In this case the condition that $\lambda+i \omega$ is in the resolvent set of $A$ for all real $\omega$ does not guarantee existence of subspaces with bounds on the semigroup as presented in Section 2 therefore we have to postulate them. With these changes and $\alpha=0$ (hence $X^{\alpha}=X,\|\cdot\|_{\alpha}=\|\cdot\|$ ), the results in Sections 3 and 4 apply unchanged. For the sake of clarity let me state explicitly all assumptions needed in this case.

(V1) $X$ is a complex Banach space.

(V2) There exists $M_{0} \in(0, \infty)$ such that if $f \in C(\mathbb{R} \backslash\{0\}, X)$ and $\|f(\cdot)\| \in L^{1}(\mathbb{R}) \cap L^{2}(\mathbb{R})$, then

$$
\int_{-\infty}^{\infty}\|f(t)\|^{2} d t \leqslant M_{0} \int_{-\infty}^{\infty}\|\hat{f}(\omega)\|^{2} d \omega \leqslant M_{0}^{2} \int_{-\infty}^{\infty}\|f(t)\|^{2} d t
$$


where

$$
\hat{f}(\omega)=\frac{1}{\sqrt{2 \pi}} \int_{-\infty}^{\infty} e^{-i \omega t} f(t) d t \quad \text { for } \quad \omega \in \mathbb{R}
$$

(V3) $-A$ is the generator of a strongly continuous semigroup $e^{-A t}$, $t \geqslant 0$, on $X$.

(V4) $X=X_{1} \oplus X_{2}$ with $e^{-A t} X_{1}=X_{1}, e^{-A t} X_{2} \subset X_{2}$ for $t \geqslant 0$, and there exist $M_{1}<\infty$ and $-\infty<\lambda_{1}<\lambda_{2}<\infty$ such that

$$
\begin{array}{rlrl}
\left\|e^{-A t} x\right\| & \leqslant M_{1} e^{-\lambda_{2} t}\|x\| & \text { for } t \geqslant 0, \quad x \in X_{2} \\
\|x\| \leqslant M_{1} e^{\lambda_{1} t}\left\|e^{-A t} x\right\| & \text { for } t \geqslant 0, \quad x \in X_{1}
\end{array}
$$

(V5) $F: \mathbb{R} \times X \rightarrow X$ is continuous and such that for some $B_{1}, \ldots, B_{m} \in \mathscr{B}(X)$, we have that

$$
\|F(t, x)-F(t, y)\| \leqslant \sum_{j=1}^{m}\left\|B_{j}(x-y)\right\| \quad \text { for } \quad t \in \mathbb{R}, \quad x, y \in X
$$

(V6) $\lambda \in\left(\lambda_{1}, \lambda_{2}\right)$ and $\int_{-\infty}^{0}\left\|e^{\mu t} F(t, 0)\right\|^{2} d t<\infty$ for some $\mu<\lambda$.

(V7) $M_{0} \sum_{j=1}^{m} \sup _{\omega \in \mathbb{R}}\left\|B_{j}(A-\lambda-i \omega)^{-1}\right\|<1$.

Observe that (V4) implies that there exist projections $P_{1}, P_{2} \in \mathscr{B}(X)$ such that $P_{i} X=X_{i}, P_{i} e^{-A t}=e^{-A t} P_{i}$ for $i=1,2, t \geqslant 0$, and $P_{1}+P_{2}=I$. (V4) also implies that $e^{-A t}$ is invertible on $X_{1}$, hence, it can be extended to a strongly continuous group defined by

$$
\mathscr{B}\left(X_{1}\right) \ni e^{-A_{1} t}= \begin{cases}e^{-A t} & \text { for } t \geqslant 0 \\ \left(e^{A t}\right)^{-1} & \text { for } t<0\end{cases}
$$

For $z \in \mathbb{C}$ with $\lambda_{1}<\operatorname{Re} z<\lambda_{2}$, one can easily show that $z$ is in the resolvent set of $A$ and that, for all $x \in X$,

$$
\begin{aligned}
& (A-z)^{-1} P_{1} x=-\int_{-\infty}^{0} e^{-A_{1} t} e^{z t} P_{1} x d t \\
& (A-z)^{-1} P_{2} x=\int_{0}^{\infty} e^{-A t} e^{z t} P_{2} x d t \\
& \left\|(A-z)^{-1}\right\| \leqslant M_{1}\left(\frac{\left\|P_{1}\right\|}{\operatorname{Re} z-\lambda_{1}}+\frac{\left\|P_{2}\right\|}{\lambda_{2}-\operatorname{Re} z}\right)
\end{aligned}
$$

As in Section 2 let $B_{0}=l \cdot I$ with $l \in(0, \infty)$ so small that

$$
\rho(\lambda) \equiv M_{0} \sum_{j=0}^{m} c_{j}(\lambda)<1
$$


where

$$
c_{j}(\lambda)=\sup _{\omega \in \mathbb{R}}\left\|B_{j}(A-\lambda-i \omega)^{-1}\right\| \quad \text { for } \quad j=0,1, \ldots, m
$$

(V6), (V7), and $\rho(\lambda)<1$ also remain valid if $\lambda$ is replaced with $\tilde{\lambda}$, provided that $|\lambda-\widetilde{\lambda}|$ is small enough. Note that

$$
\|F(t, x)-F(t, y)\| \leqslant L\|x-y\| \quad \text { for } \quad t \in \mathbb{R}, \quad x, y \in X
$$

where

$$
L=\sum_{j=0}^{m}\left\|B_{j}\right\|
$$

Now $\alpha=0, X^{\alpha}=X,\|\cdot\|_{\alpha}=\|\cdot\|$. Clearly, one can find $a<\lambda_{1}$ and $M<\infty$ so that the bounds (2.1) hold.

Theorem 5.1. In Sections 3 and 4 everything remains valid under the above assumptions (V1)-(V7) and with the above notation.

Observe also that proofs of Lemmas 3.3, 3.5, and 4.3 can be simplified when $\alpha<1 / 2$.

6. Examples. The following examples are presented for comparison purposes.

Example 6.1. Assume that $A$ is a self-adjoint operator in a Hilbert space $X$ and that

the spectrum of $A$ is contained in $\left(a, \lambda_{1}\right] \cup\left[\lambda_{2}, \infty\right)$

for some $-\infty<a<\lambda_{1}<\lambda_{2}<\infty$. $A$ can have a continunous spectrum. Observe that if $\alpha \in[0,1)$ and $\lambda \in\left(\lambda_{1}, \lambda_{2}\right)$, then

$$
\sup _{\omega \in \mathbb{R}}\left\|(A-a)^{\alpha}(A-\lambda-i \omega)^{-1}\right\| \leqslant \max \left\{\frac{\left(\lambda_{1}-a\right)^{\alpha}}{\lambda-\lambda_{1}}, \frac{\left(\lambda_{2}-a\right)^{\alpha}}{\lambda_{2}-\lambda}\right\}
$$

Fix $\alpha \in[0,1)$ and assume that $F: \mathbb{R} \times X^{\alpha} \rightarrow X$ is continuous and that for some $L \in[0, \infty)$,

$$
\|F(t, x)-F(t, y)\| \leqslant L\left\|(A-a)^{\alpha}(x-y)\right\| \quad \text { for } t \in \mathbb{R}, x, y \in \mathscr{D}\left((A-a)^{\alpha}\right)=X^{\alpha}
$$

Using (6.1) with $\lambda \in\left(\lambda_{1}, \lambda_{2}\right)$ that minimizes the right-hand side of $(6.1)$, we see that all assumptions $(\mathbf{H} 1)-(\mathbf{H} 7)$ are satisfied if

$$
\int_{-\infty}^{0}\left\|e^{\lambda_{1} t} F(t, 0)\right\|^{2} d t<\infty
$$


and

$$
L\left(\left(\lambda_{2}-a\right)^{\alpha}+\left(\lambda_{1}-a\right)^{\alpha}\right)<\lambda_{2}-\lambda_{1}
$$

In the literature the conditions corresponding to (6.2) are much more involved (see (5.1) of Chow and Lu [3], (5.3) of Foias et al. [4], pp. 143-150 of Henry [6], p. 423 of Temam [9]). Their expressions become singular as $\alpha \rightarrow 1(\alpha \leqslant 1 / 2$ of Temam [9]. Mowever, it was known [8] that if $\alpha=0$, then (6.2) is sufficient for the existence of an inertial manifold when the spectrum of $A$ consists of eigenvalues only-which is assumed also by Foias et al. [4] and Temam [9]. No assumptions on the range of $F$ are made here, however, if one has that $F: X^{\alpha+\beta} \rightarrow X^{\beta}$ for some $\beta>0$, then one may want to use $X^{\beta}$ instead of $X$ for the basic space.

Example 6.2. Consider

$$
\begin{aligned}
u_{t} & =u_{x x}+f\left(x, t, u, u_{x}\right), \quad 0<x<\pi, \quad t>0 \\
u(0, t) & =u(\pi, t)=0
\end{aligned}
$$

where $f:[0, \pi) \times \mathbb{R} \times \mathbb{C} \times \mathbb{C} \rightarrow \mathbb{C}$ is continuous and such that, for some $L_{1}<\infty, L_{2}<\infty$, we have that for all values of arguments,

$$
\left|f\left(x, t, z_{1}, z_{2}\right)-f\left(x, t, s_{1}, s_{2}\right)\right| \leqslant L_{1}\left|z_{1}-s_{1}\right|+L_{2}\left|z_{2}-s_{2}\right|
$$

Let $X=L^{2}(0, \pi), \quad A u=-u^{\prime \prime} \quad$ for $u \in H_{0}^{1}(0, \pi) \cap H^{2}(0, \pi), \quad B_{1} u=L_{1} u$, $B_{2} u=L_{2} u^{\prime}$ for $y \in H^{1}(0, \pi), \alpha \in[1 / 2,1), \lambda=\left(n^{2}+(n+1)^{2}\right) / 2$. By using (6.1), (H7) becomes

$$
\frac{L_{1}}{n+1 / 2}+L_{2} \frac{n+1}{n+1 / 2}<1
$$

Thus if $L_{2}<1$ and

$$
\int_{-\infty}^{0} d t \int_{0}^{\pi} d x\left|e^{\mu t} f(x, t, 0,0)\right|^{2}<\infty
$$

for some real $\mu$, then all assumption $(\mathrm{H} 1)-(\mathrm{H} 7)$ can be satisfied by choosing $n$ large enough. Existence of an invariant manifold for sufficiently small $L_{2}$ has been shown by Brunovsky and Terescak (1989).

\section{ACKNOWLEDGMENTS}

It is my pleasure to thank Professor Pavol Brunovsky for many indispensable discussions and a critical reading of the manuscript. I also wish to thank Professor E. Fabes for bringing the work of Vagi [10] to 
may attention and to Professors S.-N. Chow, R. A. Johnson, K. Lu, J. Mallet-Paret, H. Matano, and H. Weinberger for many illuminating discussions.

\section{REFERENCES}

1. Babin, A. V., and Vishik, M. I. (1986). Unstable invariant sets of semigroups of nonlinear operators and their perturbations. Russ. Math. Surv. 41, 1-41.

2. Brunovský, P., and Tereščák, I. (1991). Regularity of invariant manifolds. J. Diff. Eq. 3, 313-337.

3. Chow, S.-N., and Lu, K. (1988). Invariant manifolds for flows in Banach spaces. J. Diff. Eq. 74, $285-317$.

4. Foias, C., Sell, G. R., and Titi, E. S. (1989). Exponential tracking and approximation of inertial manifolds for dissipative nonlinear equations. J. Dynam. Diff. Eq. 1, 199-244.

5. Hale, J. K. (1988). Asymptotic Behavior of Dissipative Systems, Math. Surveys Monogr. 25, Am. Math. Soc., Providence.

6. Henry, D. (1981). Geometric Theory of Semilinear Parabolic Equations, Lecture Notes in Math., 840, Springer-Verlag, New York.

7. Mallet-Paret, J., and Sell, G. R. (1988). Inertial manifolds for reaction diffusion equations in higher space dimensions. J. Am. Math. Soc. 1, 805-866.

8. Matano, H. (1989). Personal communication.

9. Temam, R. (1988). Infinite-Dimensional Dynamical Systems in Mechanics and Physics, Springer-Verlag, New York.

10. Vagi, S. (1969). A remark on Plancherel's theorem for Banach space valued functions. Ann. Scuola Norm. Sup. Pisa 23, 305-315. 